\title{
NUDT15 Variants Cause Hematopoietic Toxicity with Low 6-TGN Levels in Children with Acute Lymphoblastic Leukemia
}

\section{Eun Sang Yi, MD ${ }^{1,2}$ \\ Young Bae Choi, MD \\ Rihwa Choi, MD ${ }^{4}$ \\ Na Hee Lee, MD \\ Ji Won Lee, MD, PhD 1 \\ Keon Hee Yoo, MD, PhD' \\ Ki Woong Sung, MD, PhD' \\ Soo-Youn Lee, MD, PhD ${ }^{4}$ \\ Hong Hoe Koo, MD, PhD}

\begin{abstract}
${ }^{1}$ Department of Pediatrics, Samsung Medical Center, Sungkyunkwan University School of Medicine, Seoul, ${ }^{2}$ Department of Pediatrics, Korea University Guro Hospital, Korea University College of Medicine, Seoul, ${ }^{3}$ Department of Pediatrics, Chung-Ang University Hospital, Seoul, ${ }^{4}$ Department of Laboratory Medicine and Genetics, Samsung Medical Center, Sungkyunkwan University School of Medicine, Seoul, ${ }^{5}$ Department of Pediatrics, CHA Bundang Medical Center, CHA University, Seongnam, Korea
\end{abstract}

Correspondence: Hong-Hoe Koo, MD, PhD Department of Pediatrics, Samsung Medical Center, Sungkyunkwan University School of Medicine, 81 Irwon-ro, Gangnam-gu, Seoul 06351, Korea

Tel: 82-2-3410-3539

Fax: 82-2-3410-0043

E-mail: hhkoo@skku.edu

Co-correspondence: Soo-Youn Lee, MD, PhD Department of Laboratory Medicine and Genetics, Samsung Medical Center, Sungkyunkwan University School of Medicine, 81 Irwon-ro, Gangnam-gu, Seoul 06351, Korea Tel: 82-2-3410-1834

Fax: 82-2-3410-2719

E-mail: suddenbz@skku.edu

Received June 12, 2017

Accepted September 6, 2017

Published Online September 13, 2017

${ }^{*}$ Eun Sang Yi and Young Bae Choi contributed equally to this work.

\section{Purpose}

We aimed to identify the impact of NUDT15 variants on thiopurine intolerance and 6-thioguanine nucleotide (6-TGN) levels in Korean children with acute lymphoblastic leukemia (ALL).

\section{Materials and Methods}

Genotyping of NUDT15 was tested in 258 patients with ALL registered at Samsung Medical Center. Patients were classified into normal-activity (wild-type), intermediate-activity (heterozygous variant), and low-activity groups (homozygous or compound heterozygous variant). Clinical and laboratory features during the first year of maintenance therapy were investigated.

\section{Results}

A total of 182 patients were included in the final analysis. There were five $(2.7 \%), 46$ (25.3\%), and 131 (72.0\%) patients in low-, intermediate-, and normal-activity groups, respectively. The lowest 6-mercaptopurine (6-MP) dose $\left(\mathrm{mg} / \mathrm{m}^{2} /\right.$ day) was administered to the low-activity group (low-activity group 7.5 vs. intermediate-activity group 24.4 vs. normalactivity group 31.1, $p<0.01$ ) from three months to a year after beginning maintenance therapy. The low-activity group experienced the longest duration of therapy interruption during the first year (low-activity group 169 days vs. intermediate-activity group 30 days vs. normal-activity group 16 days, $p<0.01$ ). They also showed the lowest blood cell counts and had a longer duration of leukopenia (low-activity group 131 days vs. intermediate-activity group 92 days vs. normal-activity group 59 days, $p<0.01$ ). 6-TGN level and its ratio to 6-MP dose were lowest in the low-activity group.

\section{Conclusion}

NUDT15 variants cause hematopoietic toxicity with low 6-TGN levels. NUDT15 genotyping should be conducted before administering thiopurine, and dose adjustments require caution regardless of 6-TGN levels.

\author{
Key words \\ NUDT15, Leukemia, 6-Thioguanylic acid, Thiopurine, \\ 6-Mercaptopurine
}




\section{Introduction}

Thiopurines, such as 6-mercaptopurine (6-MP) are widely used for the treatment of childhood acute lymphoblastic leukemia (ALL). The optimized use of 6-MP has shown a remarkable improvement in survival [1,2]. As per treatment protocols, children with ALL are exposed to thiopurines on a daily basis for up to two or three years during maintenance therapy $[3,4]$.

Hematopoietic toxicity is one of the most important doselimiting adverse effects of thiopurines [5,6]. East Asians are the ethnic group most affected by thiopurines $[7,8]$. Patients often experience serious bone marrow suppression, infectious complications, and interruptions in therapy. Low systemic exposure to thiopurines affects prognosis $[9,10]$. Lower 6-MP dose intensity is primarily due to a missed period of therapy, not reductions in the daily dose [10]. In a Children's Oncology Group (COG) study, 6-MP non-adherence and high intra-individual variability in thiopurine metabolites were associated with relapse risk [11]. Thiopurine S-methyltransferase (TPMT) variants are well-established genetic determinants for cytotoxicity of thiopurines [12,13]. Dose adjustment guidelines based on 6-thioguanine nucleotide (6-TGN) levels have enabled patients with TPMT variants to minimize hematopoietic toxicity while maintaining its therapeutic effect $[14,15]$

Nucleoside diphosphate-linked moiety X-type motif 15 (NUDT15) has recently been discovered to be associated with hematopoietic toxicity, initially in patients with inflammatory bowel disease [16]. A negative effect of NUDT15 variants was also revealed in children with ALL by previous reports that mainly focused on the less tolerated doses of 6-MP in patients with NUDT15 variants [17-19]. The hypothesis about the mechanism of NUDT15 variants suggested that the NUDT15 protein encoded by NUDT15 might prevent 6-TGN, the active metabolite of thiopurine, from being incorporated into the DNA [20]. This hypothesis has been supported by Moriyama et al. [18], who recently reported a high thioguanosine triphosphate (TGTP)/monophosphate thioguanosine nucleotide (TGMP) ratio in a NUDT15 knockdown cell line, and high DNA-incorporated thioguanine (DNA-TG) levels in children with ALL with NUDT15 variants. Contrary to TPMT variants, there is poor information about thiopurine dose alteration for patients with NUDT15 variants. Considering the proposed hypothesis about the NUDT15 protein, NUDT15 variants are assumed to cause cytotoxicity independent of the 6-TGN levels; therefore, the same guidelines that apply to TPMT variants would not be applicable to patients with NUDT15 variants. This study aimed to analyze the levels of 6-TGN, which is widely used in clinics, according to NUDT15 variants.
In addition, this study intended to analyze hematopoietic toxicity based on NUDT15 diplotypes in Korean children with ALL more comprehensively. All six discovered haplotypes were screened. We collected clinical and laboratory information of patients during their first year of maintenance therapy because previous reports seemed to use limited indices for measuring hematopoietic toxicity or thiopurine intolerance $[17,18]$.

\section{Materials and Methods}

\section{Study patients and NUDT15 genotyping}

NUDT15 genotyping was retrospectively conducted in 258 children with ALL who underwent TPMT genotyping at Samsung Medical Center in Korea for research purposes from 2004 to 2015 (IRB file No. SMC 2012-04-106-014). Genomic DNA was extracted from whole blood using Wizard genomic DNA purification kit according to the manufacturer's instructions (Promega, Madison, WI) [21]. Exons 1 and 3, and their intron-exon boundaries of NUDT15 were amplified by polymerase chain reaction (PCR) using primers designed by our institute. For exon 3, PCR was performed using a GeneAmp PCR system 9700 thermal cycler (Biometra, Applied Biosystems Life Technologies, Foster City, CA) with 35 cycles at $94^{\circ} \mathrm{C}$ for 20 seconds, $60^{\circ} \mathrm{C}$ for 30 seconds, and $72^{\circ} \mathrm{C}$ for 40 seconds. An Eppendorf pro $\mathrm{S}$ (Eppendorf) was used for exon 1 with 50 cycles at $95^{\circ} \mathrm{C}$ for 20 seconds, $62^{\circ} \mathrm{C}$ for 30 seconds, and $72^{\circ} \mathrm{C}$ for 40 seconds. Five microliters of the amplicon for both exons was treated with $10 \mathrm{U}$ exonuclease I (USB Corp., Cleveland, $\mathrm{OH}$ ) at $37^{\circ} \mathrm{C}$ for 15 minutes, and then incubated at $80^{\circ} \mathrm{C}$ for 15 minutes to inactivate the enzyme. Cycle sequencing was performed using Big Dye Terminator Cycle Sequencing Ready Reaction kits (Applied Biosystems) in an ABI 3130xl Genetic Analyzer (Applied Biosystems). Direct sequencing of exons 1 and 3 of NUDT15 (NCBI reference sequence NM_018283.3) was performed.

Based on previous reports that demonstrated a decreased NUDT15 activity and an increased risk of hematopoietic toxicity with a number of variant alleles [18], study patients were classified into three groups according to their NUDT15 diplotype: normal-activity group (wild-type), intermediateactivity group (heterozygous variant), or low-activity group (homozygous or compound heterozygous variant). Patient demographics at diagnosis and clinical features and laboratory results during the first year of maintenance therapy were obtained from patients who had received maintenance therapy, including 6-MP at Samsung Medical Center via retrospective chart review. Patents with TPMT variants were 
excluded from the analysis.

\section{6-TGN assays}

Since May 2009, thiopurine metabolites assay has been studied using whole blood from patients initially at 14 th day after beginning maintenance therapy and at 14th day after dose alteration, until the white blood cell (WBC) count and 6-TGN levels were within the target range. It was performed only when patients had taken the same dose of 6-MP for at least 14 days. Red blood cell (RBC) levels of 6-TGN were measured using a liquid chromatography-tandem mass spectrometry method [22]. Metabolite levels were normalized to an RBC count of $8 \times 10^{8}$. The limit of quantification was $0.1 \mu \mathrm{mol} / \mathrm{L}$ for $6-\mathrm{TGN}$, corresponding to quantification limits of approximately 10 pmol per $8 \times 10^{8} \mathrm{RBC}$ for 6-TGN [23].

\section{ALL risk stratification and treatment schedule}

Patients $\geq 10$ years or $<12$ months of age, or who had an initial WBC count $\geq 50 \times 10^{9} / \mathrm{L}$ were considered as high-risk patients at initial diagnosis. All others were considered as standard-risk patients. Patients with any high-risk characteristics received more intensified chemotherapy during induction and consolidation therapy compared to standard-risk patients. Patients with at least one of the characteristics, including an initial WBC count of $\geq 200 \times 10^{9} / \mathrm{L}$, positive bcr/abl rearrangement, hypodiploidy under 45 chromosomes by cytogenetics, $t(4 ; 11)$, or remission failure received allogeneic stem cell transplantation when a suitable donor was available.

Maintenance therapy consisted of daily oral 6-MP (50 $\left.\mathrm{mg} / \mathrm{m}^{2}\right)$, weekly oral methotrexate $\left(15 \mathrm{mg} / \mathrm{m}^{2}\right)$, oral pred- nisolone $\left(40 \mathrm{mg} / \mathrm{m}^{2}\right)$ for 5 days, intravenous vincristine $(1.5$ $\mathrm{mg} / \mathrm{m}^{2}$ ) every 4 weeks, and intrathecal triple therapy (methotrexate, cytarabine, and hydrocortisone) every 12 weeks. 6-MP and methotrexate doses were altered at the discretion of pediatric oncologists based on the complete blood count $(\mathrm{CBC})$ and 6-TGN levels. When the WBC count was consecutively out of target range $\left(1.5-3.0 \times 10^{9} / \mathrm{L}\right)$, the 6 -MP dose was adjusted if 6-TGN levels were out of therapeutic range (235-450 $\left.\mathrm{pmol} / 8 \times 10^{8} \mathrm{RBC}\right)$, or the methotrexate dose was altered if 6 -TGN levels were within the therapeutic range. Treatment was interrupted if patients developed significant hematopoietic toxicity (absolute neutrophil count $<0.5 \times 10^{9} / \mathrm{L}$ or platelet count $<50 \times 10^{9} / \mathrm{L}$ ) or a serious infectious event.

\section{Statistical analysis}

Clinical features and laboratory results at initial diagnosis and during the first year of maintenance therapy were compared across groups using chi-square and Fisher exact tests for categorical variables, and one way analysis of variance (ANOVA) or Kruskal-Wallis test along with Tukey test using ranks for continuous variables. Because 6-MP doses were frequently altered during the initial period of maintenance therapy, 6-MP doses during the initial 3 months were excluded from analysis for comparing the administered 6-MP doses between the groups. Consequently, 6-MP doses from 3 months to a year after maintenance therapy were compared. Correlation between the 6-TGN level and 6-MP dose was analyzed by Pearson's correlation analysis. Subsequently, the ratio of 6-TGN level to 6-MP dose was compared among the groups. All statistical analyses were performed using SPSS ver. 22.0 (IBM Corp., Armonk, NY).

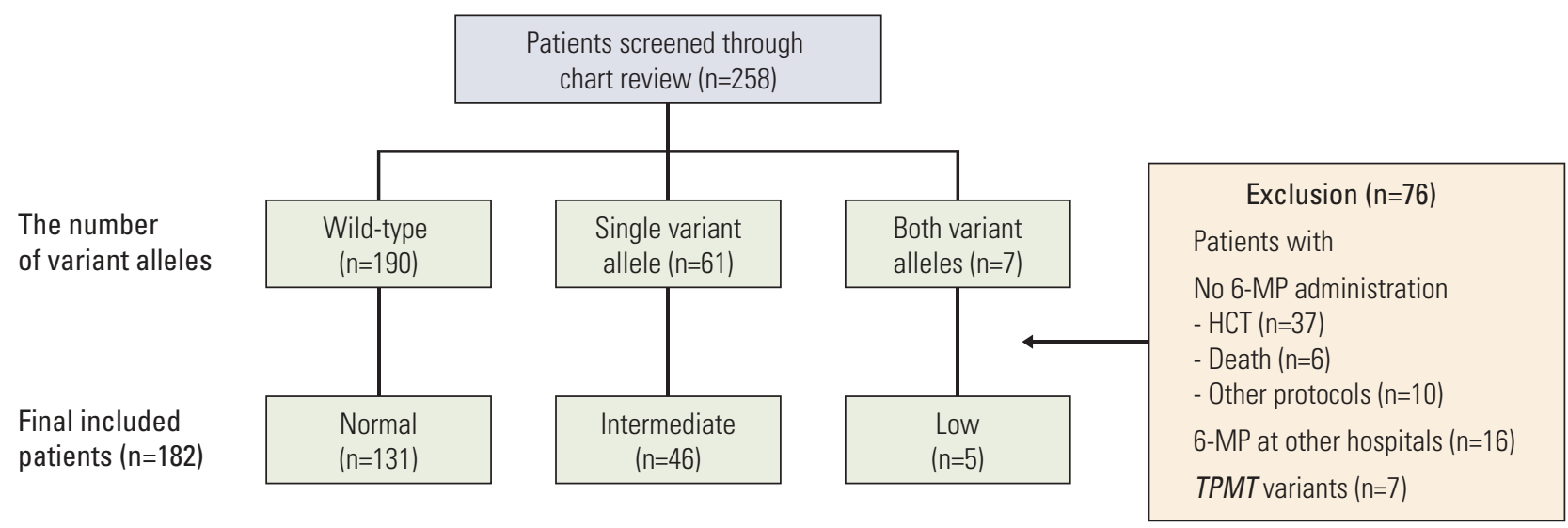

Fig. 1. Study population. Patients were classified into normal-, intermediate-, low-activity groups according to the number of variant alleles. 6-MP, 6-mercaptopurine; HCT, hematopoietic stem cell transplantation; TPMT, thiopurine S-methyltransferase. 
Table 1. Allele frequency in the study population

\begin{tabular}{lllr} 
NUDT15 allele & \multicolumn{1}{c}{ Genetic variant } & \multicolumn{1}{c}{ Protein variant } & \multicolumn{1}{c}{ Proportion (\%) } \\
${ }^{*} 1$ & Wild-type & Wild-type & 85.5 \\
${ }^{*} 2$ & c.[36_37insGGAGTC; c.415C >T] & p.Val18_Val19insGlyVal/p.Arg139Cys & 5.2 \\
${ }^{*} 3$ & c.415C>T & p.Arg139Cys & 7.9 \\
$* 4$ & c.416G>A & p.Arg139His & 0.2 \\
${ }^{*} 5$ & c.52G>A & p.Val18Ile & 0.8 \\
$* 6$ & c.36_37insGGAGTC & p.Val18_Val19insGlyVal & 0.4 \\
\hline
\end{tabular}

\section{Ethical statement}

The study was approved by the Institutional Review Board of Samsung Medical center (IRB No. SMC 2017-01-015-001) and performed in accordance with the principles of the Declaration of Helsinki. The informed consent was waived.

\section{Results}

\section{Characteristics of study patients}

NUDT15 genotyping was conducted for a total of 258 patients (Fig. 1). According to their NUDT15 diplotype, 190 patients were wild-type $(73.6 \%), 61$ patients had a single variant allele $(23.6 \%)$, and seven patients had variants on both alleles $(2.7 \%)$. Allele frequency and composition of NUDT15 diplotypes in the study population are described in Tables 1 and 2, respectively. Regarding allele frequency, NUDT15*1 (wild-type) was the most common (85.5\%), followed by *3 $(7.9 \%)$ and ${ }^{*} 2(5.2 \%)$. Regarding diplotype frequency, NUDT15*1/*3 $(12.8 \%)$ was the most common variant, followed by NUDT15*1/*2 (8.5\%). All six previously known haplotypes were observed in this study.

Of the total study population, 76 patients were excluded from the analysis. Forty-three patients stopped maintenance therapy for hematopoietic stem cell transplantation $(n=37)$ or due to death during induction or consolidation therapy $(n=6)$. Sixteen patients received maintenance therapy at other hospitals. Ten patients received maintenance therapy without daily 6-MP, according to adult ALL treatment protocols $(n=5)$ or other protocols $(n=5)$. Seven patients were TPMT heterozygous. A total of 182 patients were included in the final analysis. To analyze hematopoietic toxicity based on NUDT15 diplotypes, patients were classified into three groups: normal-activity ( $\mathrm{n}=131)$, intermediate-activity $(\mathrm{n}=46)$, and low-activity $(n=5)$ groups. Characteristics of the study patients are summarized in Table 3 . The three groups were
Table 2. Composition of NUDT15 diplotypes in the study population

\begin{tabular}{lrr} 
Diplotype & No. of patients $(\mathbf{n}=258)$ & Proportion $(\%)$ \\
${ }^{*} 1 /{ }^{*} 1$ & 190 & 73.6 \\
${ }^{*} 1 /{ }^{*} 2$ & 22 & 8.5 \\
${ }^{*} 1 /{ }^{*} 3$ & 33 & 12.8 \\
${ }^{*} 1 /{ }^{*} 5$ & 4 & 1.6 \\
${ }^{*} 1 /{ }^{*} 6$ & 2 & 0.8 \\
${ }^{*} 2 /{ }^{*} 3$ & 4 & 1.6 \\
${ }^{*} 2 /{ }^{*} 4$ & 1 & 0.4 \\
${ }^{*} 3 /{ }^{*} 3$ & 2 & 0.8 \\
\hline
\end{tabular}

similar with respect to demographic characteristics, including sex, age, ALL risk group, initial WBC counts, cytogenetic results, immunophenotype, and rapid early response, except central nervous system disease at diagnosis.

\section{Hematopoietic toxicity according to NUDT15 diplotypes}

Fig. 2 shows the daily 6-MP doses administered to patients, duration of therapy interruption, and proportion of patients who experienced febrile neutropenia. Daily 6-MP dose was defined as the average daily dose of 6-MP administered from three months to a year after maintenance therapy. The 6-MP dose during therapy interruption was considered to be zero. With accretion of risk by NUDT15 diplotype, the daily 6-MP doses were decreased and therapy interruption days were increased. Mean daily 6-MP doses ( \pm standard deviation [SD]) were $31.1 \pm 8.7 \mathrm{mg} / \mathrm{m}^{2}$ in the normal-activity group, $24.4 \pm 7.7$ $\mathrm{mg} / \mathrm{m}^{2}$ in the intermediate-activity group, and $7.5 \pm 3.0 \mathrm{mg} / \mathrm{m}^{2}$ in the low-activity group $(\mathrm{p}<0.01)$. The mean number of interrupted days in the first year of maintenance therapy $( \pm S D)$ were $15.5 \pm 17.8,30.3 \pm 29.4$, and $168.8 \pm 50.1$ days in the normal-, intermediate-, and low-activity groups, respectively $(\mathrm{p}<0.01)$. Febrile neutropenia was most frequent in the lowactivity group during the first year of maintenance therapy (normal-activity group $14.6 \%$ vs. intermediate-activity group $15.2 \%$ vs. low-activity group $80 \%, \mathrm{p}<0.01$ ). 
Table 3. Characteristics of the study patients and composition of diplotypes

\begin{tabular}{|c|c|c|c|c|c|}
\hline & $\begin{array}{l}\text { All patients } \\
\qquad(\mathrm{n}=182)\end{array}$ & $\begin{array}{l}\text { Normal- } \\
\text { activity group } \\
(\mathbf{n}=131)\end{array}$ & $\begin{array}{l}\text { Intermediate- } \\
\text { activity group } \\
\quad(n=46)\end{array}$ & $\begin{array}{c}\text { Low- } \\
\text { activity group } \\
(n=5)\end{array}$ & p-value \\
\hline \multicolumn{6}{|l|}{ Age (yr) } \\
\hline At diagnosis & $5.2(1.1-21.3)$ & $5.2(1.1-21.3)$ & $4.8(1.1-16.0)$ & $6.1(3.5-13.5)$ & 0.53 \\
\hline At maintenance & $6.0(1.9-22.0)$ & $6.0(1.9-22.0)$ & $5.7(2.0-17.2)$ & $7.1(4.6-14.8)$ & 0.52 \\
\hline \multicolumn{6}{|l|}{ Sex } \\
\hline Female & $86(47.3)$ & $61(46.6)$ & $21(45.7)$ & $4(80.0)$ & 0.33 \\
\hline Male & $96(52.7)$ & $70(53.4)$ & $25(54.3)$ & $1(20.0)$ & \\
\hline \multicolumn{6}{|l|}{ ALL risk group } \\
\hline Standard & $117(64.3)$ & $85(64.9)$ & $30(65.2)$ & $2(40.0)$ & 0.52 \\
\hline High & $65(35.7)$ & $46(35.1)$ & $16(34.8)$ & $3(60.0)$ & \\
\hline \multicolumn{6}{|c|}{ WBC counts at diagnosis $\left(\times 10^{9} / \mathrm{L}\right)$} \\
\hline$<50$ & $171(94.0)$ & $125(95.4)$ & $41(89.1)$ & $5(100)$ & 0.604 \\
\hline $50-100$ & $9(4.9)$ & $5(3.8)$ & $4(8.7)$ & - & \\
\hline$>100$ & $2(1.1)$ & $1(0.8)$ & $1(2.2)$ & - & \\
\hline \multicolumn{6}{|c|}{ Favorable cytogenetics ${ }^{a}$} \\
\hline No & $75(41.4)$ & $51(39.2)$ & $23(50.0)$ & $1(20.0)$ & 0.273 \\
\hline Yes & $106(58.6)$ & $79(60.8)$ & $23(50.0)$ & $4(80.0)$ & \\
\hline \multicolumn{6}{|c|}{ Unfavorable cytogenetics ${ }^{\text {b) }}$} \\
\hline No & $167(92.3)$ & $121(93.1)$ & $41(89.1)$ & $5(100)$ & 0.556 \\
\hline Yes & $14(7.7)$ & $9(6.9)$ & $5(10.9)$ & - & \\
\hline \multicolumn{6}{|c|}{ CNS disease at diagnosis } \\
\hline No & $166(92.2)$ & $120(93.0)$ & $43(93.5)$ & $3(60.0)$ & 0.28 \\
\hline Yes & $4(2.2)$ & $3(2.3)$ & - & $1(20.0)$ & \\
\hline Traumatic tap & $10(5.6)$ & $6(4.7)$ & $3(6.5)$ & $1(20.0)$ & \\
\hline \multicolumn{6}{|l|}{ Immunophenotype } \\
\hline $\mathrm{B}$ & $173(95.1)$ & $125(95.4)$ & $43(93.5)$ & $5(100)$ & 0.969 \\
\hline $\mathrm{T}$ & $6(3.3)$ & $4(3.1)$ & $2(4.3)$ & - & \\
\hline Others & $3(1.6)$ & $2(1.5)$ & $1(2.2)$ & - & \\
\hline \multicolumn{6}{|l|}{ RER } \\
\hline No & $66(36.9)$ & $44(34.1)$ & $20(44.4)$ & $2(40.0)$ & 0.460 \\
\hline Yes & $113(63.1)$ & $85(65.9)$ & $25(55.6)$ & $3(60.0)$ & \\
\hline
\end{tabular}

Values are presented as median (range) or number of patients (\%). Missing data for the following: favorable cytogenetics $(n=1)$, unfavorable cytogenetics $(n=1)$, CNS disease at diagnosis $(n=2)$, and RER $(n=3)$. ALL, acute lymphoblastic leukemia;


following: t(12;21), hyperdiploidy, trisomy 4 and 10, or trisomy 4,10 , and 17, b) Unfavorable cytogenetics included $\geq 1$ of the following: $t(9 ; 22), t(4 ; 11)$, iAMP21, or hypodiploidy.

The lowest values of CBC, including WBC, neutrophil, platelet, and hemoglobin are shown in Fig. 3. The low-activity group had the lowest levels of all the blood cell lineages. The means of the lowest WBC counts $( \pm \mathrm{SD})$ were $(1.358 \pm$ $0.575) \times 10^{9} / \mathrm{L}$ in the normal-activity group, $(1.114 \pm 0.295) \times 10^{9} / \mathrm{L}$ in the intermediate-activity group, and $(0.620 \pm 0.254) \times 10^{9} / \mathrm{L}$ in the low-activity group ( $p<0.01)$. The mean of the lowest absolute neutrophil count $( \pm S D)$ was lowest in the low-activity group $\left([0.040 \pm 0.037] \times 10^{9} / \mathrm{L}\right)$ compared to that in the normal-activity group $\left([0.434 \pm 0.405] \times 10^{9} / \mathrm{L}\right)$ or the intermediate- activity group $\left([0.293 \pm 0.196] \times 10^{9} / \mathrm{L}\right)$. The means of the lowest hemoglobin levels $( \pm \mathrm{SD})$ were $10.1 \pm 1.1 \mathrm{~g} / \mathrm{dL}$ in the normal-activity group, $9.8 \pm 1.1 \mathrm{~g} / \mathrm{dL}$ in the intermediate-activity group, and $7.4 \pm 1.0 \mathrm{~g} / \mathrm{dL}$ in the low-activity group $(\mathrm{p}<0.01)$. The means of the lowest platelet counts in the low-activity group $( \pm \mathrm{SD})$ were extremely low $\left([9 \pm 5] \times 10^{9} / \mathrm{L}\right)$ compared to those in the normal-activity group $\left([137 \pm 53] \times 10^{9} / \mathrm{L}\right)$ or the intermediate-activity group $\left([115 \pm 43] \times 10^{9} / \mathrm{L}\right)$. The duration of leukopenia, defined as WBC $<2 \times 10^{9} / \mathrm{L}$, increased with increasing risk of NUDT15 diplotype (Fig. 4). The low-activ- 
A
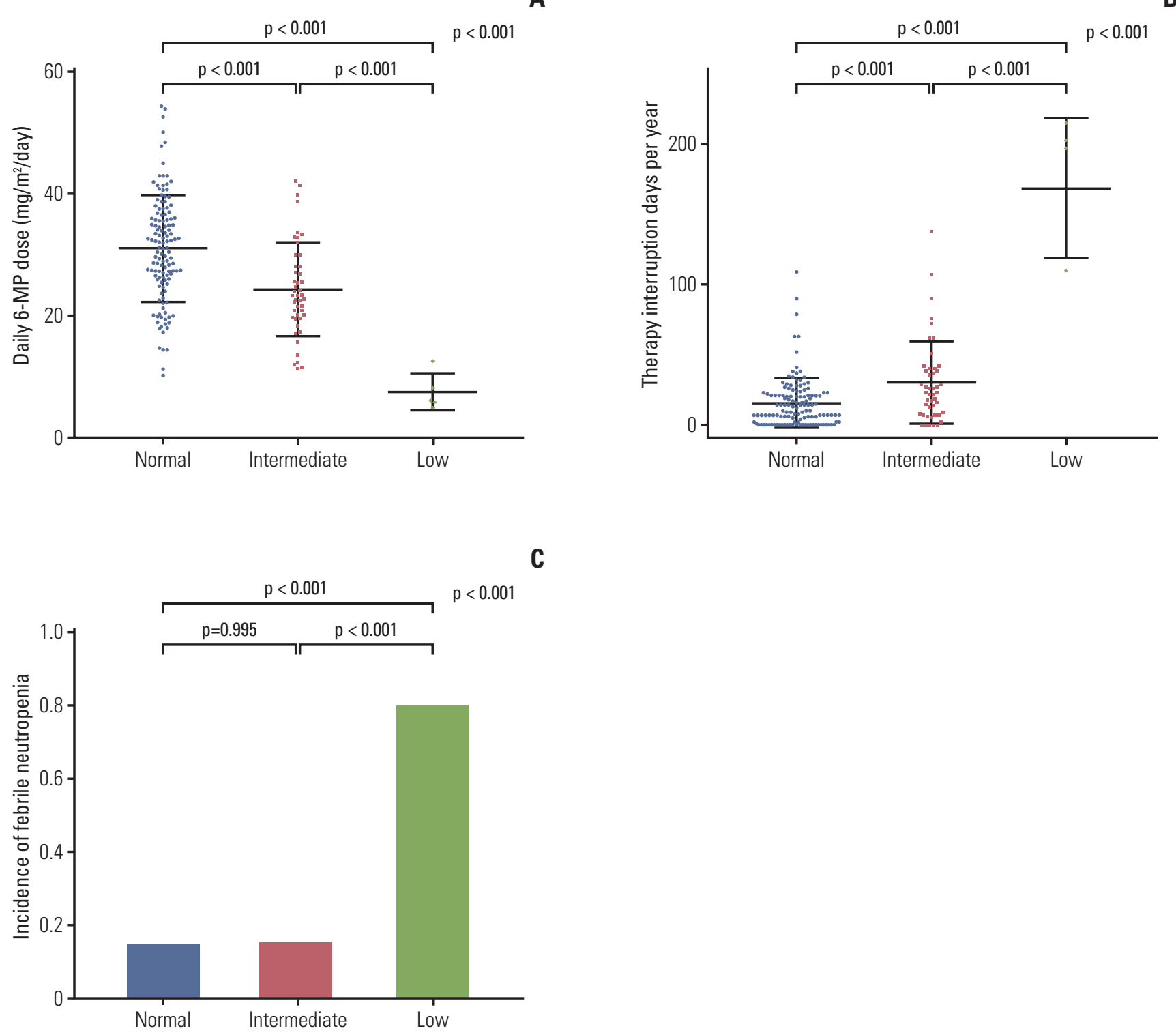

Fig. 2. Daily 6-mercaptopurine (6-MP) dose and clinical features of the groups. (A) Daily 6-MP dose. (B) Therapy interruption days. (C) Incidence of febrile neutropenia.

ity group experienced the most number of days of leukopenia (mean $\pm S D, 131 \pm 68$ days), followed by those in the intermediate-activity group ( $92 \pm 46$ days) and the normal-activity group ( $59 \pm 45$ days).

\section{6-TGN level and its ratio to 6-MP dose according to NUDT15 diplotype}

A total of 671 results of 6-TGN levels were obtained from 117 of the 182 patients ( 26 results from 5 patients in the low- activity group, 173 from 24 patients in the intermediateactivity group, and 472 from 88 patients in the normal-activity group). Fig. 5 shows the 6-TGN levels of patients whose 6-MP dose was stable for at least 14 days. The median 6-TGN levels (range) were $383(135-1,461) \mathrm{pmol} / 8 \times 10^{8} \mathrm{RBC}$ in the normal-activity group, $286(94-1,145) \mathrm{pmol} / 8 \times 10^{8} \mathrm{RBC}$ in the intermediate-activity group, and $78(5-171) \mathrm{pmol} / 8 \times 10^{8} \mathrm{RBC}$ in the low-activity group $(\mathrm{p}<0.01)$. A positive correlation was seen for 6-TGN levels in the whole study population (coefficient of correlation, $0.30 ; \mathrm{p}<0.01$ ). To assess the 6-TGN 
A
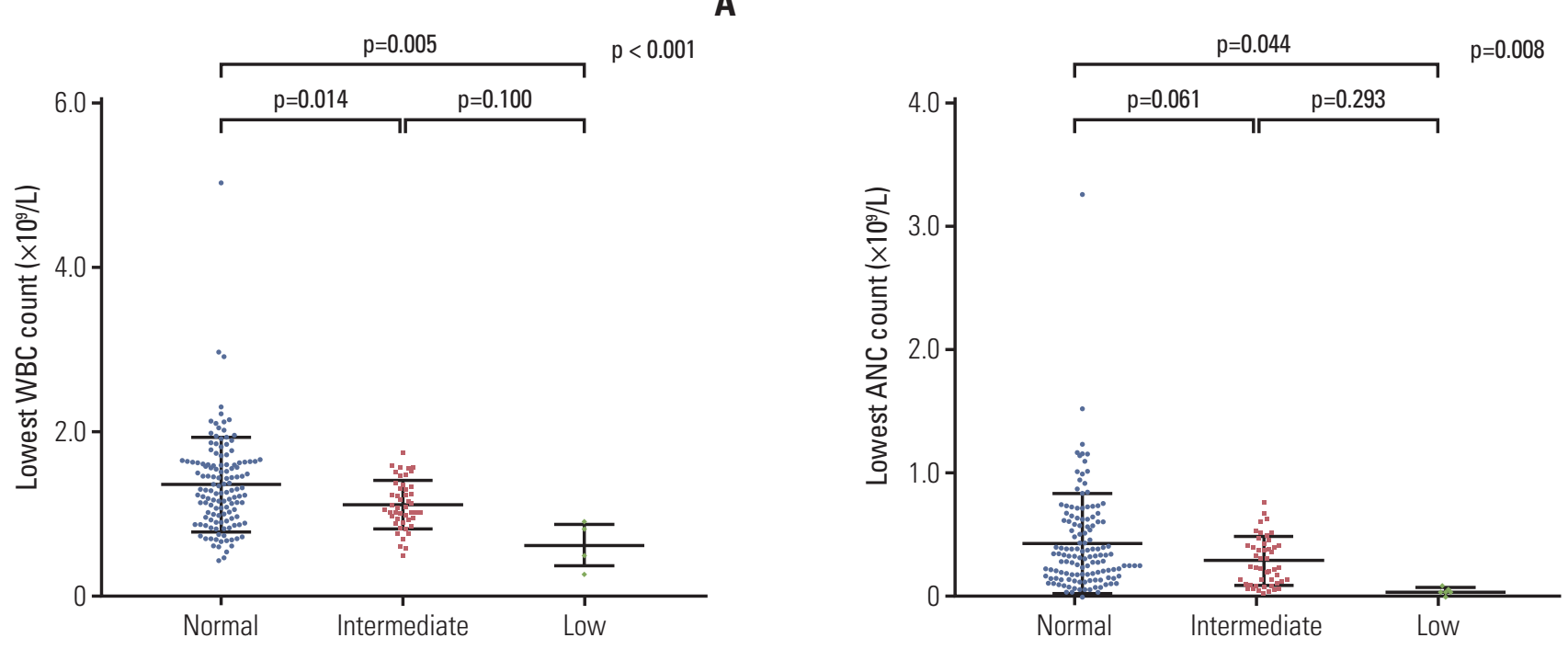

C
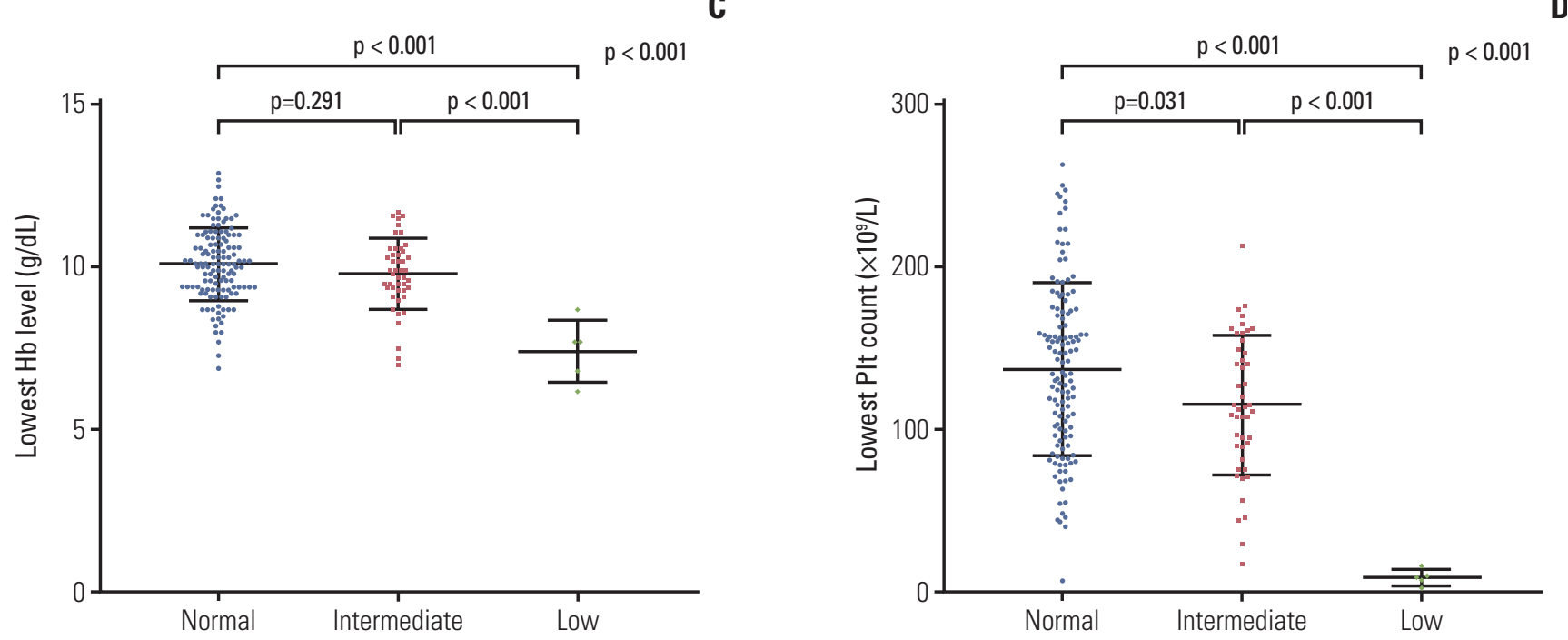

Fig. 3. Lowest level of complete blood cell counts in the groups. (A) Lowest white blood cell (WBC) counts. (B) Lowest absolute neutrophil counts (ANCs). (C) Lowest hemoglobin ( $\mathrm{Hb}$ ) levels. (D) Lowest platelet (Plt) counts.

levels with equal doses of 6-MP, the ratio of 6-TGN level to 6-MP dose was compared across groups (Fig. 6). Patients in the low-activity group had the lowest ratio (mean $\pm \mathrm{SD}$, $4.4 \pm 2.7)$ as compared to those in the intermediate-activity group (13.3 \pm 7.5$)$ and the normal-activity group (14.7 \pm 9.1$)$.

The 6-TGN levels in the low-activity group were below the alleged level associated with a higher risk of leukopenia (450 $\mathrm{pmol} / 8 \times 10^{8} \mathrm{RBC}$ ) [22]. In 26 results from the low-activity group, $10(38.5 \%)$ were performed while the patient had grade $\geq 3$ leukopenia or thrombocytopenia (platelets $<50 \times 10^{9} / \mathrm{L}$ ).

\section{Discussion}

More than a quarter of the Korean children in this study had NUDT15 variants. A lower dose of 6-MP was administered to them. They experienced therapy interruption and febrile neutropenia more frequently. They also experienced more severe cytopenia in all types of peripheral blood cells and the duration of leukopenia was longer. A lower 6-TGN level and a lower 6-TGN/6-MP ratio were also observed. 


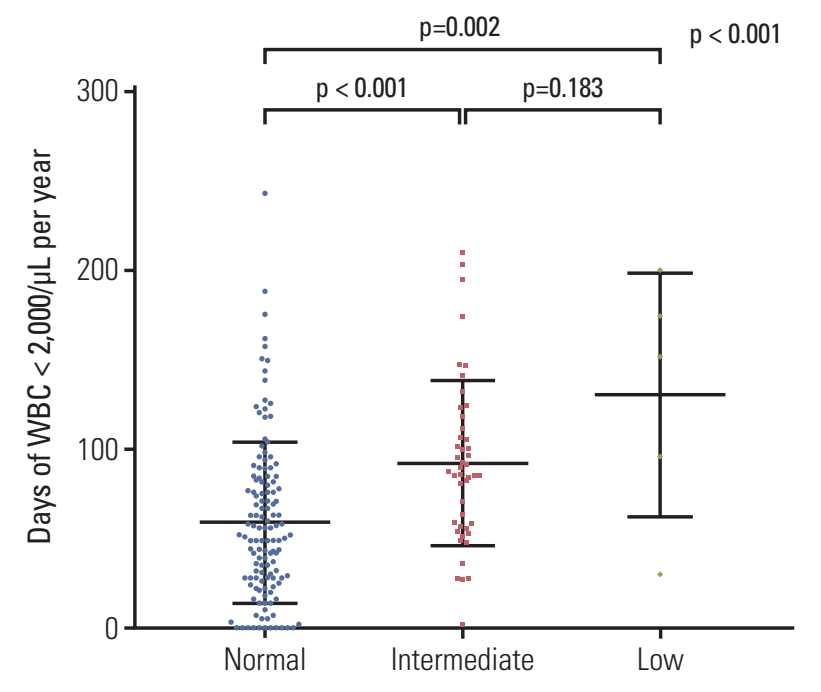

Fig. 4. Duration of leukopenia in the various groups. WBC, white blood cell.

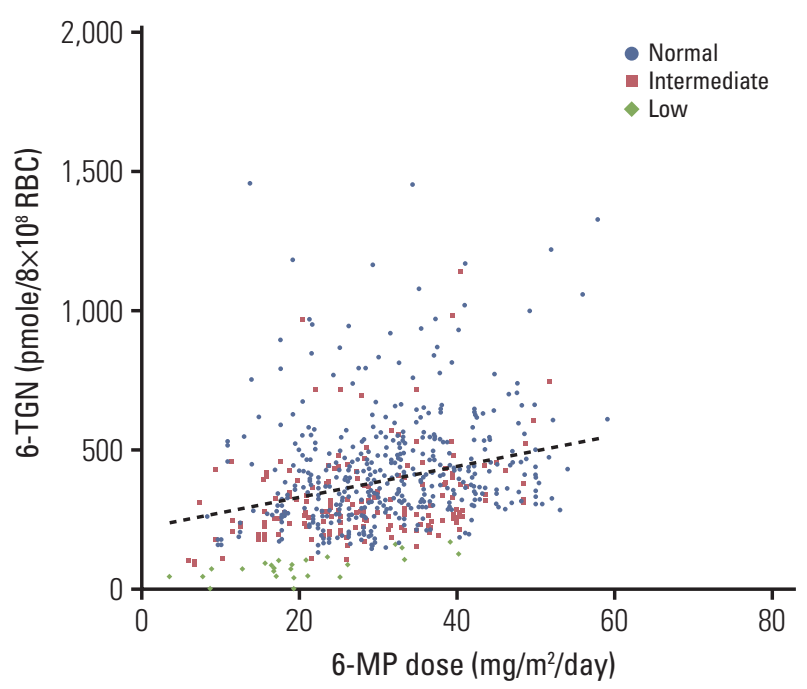

Fig. 5. 6-Thioguanine nucleotide (6-TGN) levels and 6-mercaptopurine (6-MP) doses of the study population. $\mathrm{RBC}$, red blood cell.

The lower ratio of 6-TGN level to 6-MP dose in patients with NUDT15 variants is opposite to that in patients with TPMT variants [24]. The mechanism of cytotoxicity in TPMT variants is known to be related to increased 6-TGN levels because TPMT encoded by TPMT controls the anabolic transformation of thiopurine metabolites to active metabolites and regulates the cytotoxic effect of thiopurines in children with

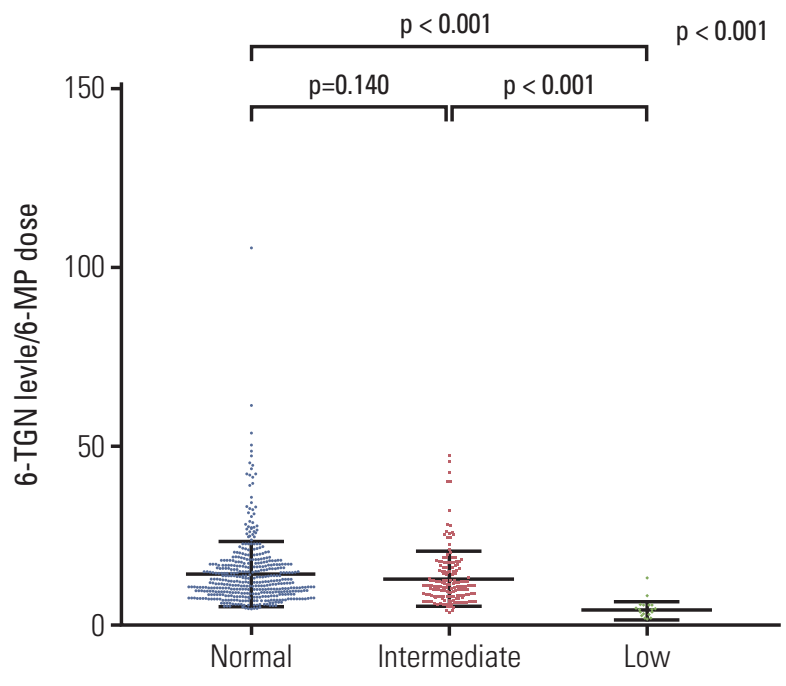

Fig. 6. Ratio of the 6-thioguanine nucleotide (6-TGN) level to 6-mercaptopurine (6-MP) dose between the groups.

ALL [25]. By phosphorylation, thiopurines are converted to TGMP, TGDP, and TGTP, and then further reduced to TdGMP, TdGDP, and TdGTP. TdGTP is directly incorporated into DNA and eventually triggers apoptosis [5]. It has been suggested that NUDT15 may prevent incorporation of 6-TGN into DNA via dephosphorylation of 6-TGN metabolites in the thiopurine metabolism pathway $[18,20,26]$. Therefore, loss-of-function NUDT15 variants confer susceptibility to thiopurine-induced cytotoxicity [16]. With the high DNATG level in patients with NUDT15 variants shown by Moriyama et al. [18], a low ratio of 6-TGN level to 6-MP dose in the low-activity group in our study seemed to be concordant with the existing hypothesis because active thiopurine metabolites could be incorporated into DNA by a defective protective mechanism resulting from loss-of-function NUDT15 variants.

Erythrocyte 6-TGN concentrations $<235 \mathrm{pmol} / 8 \times 10^{8} \mathrm{RBC}$ are considered to be associated with a greater likelihood of treatment failure, whereas 6-TGN concentrations $>450 \mathrm{pmol}$ $/ 8 \times 10^{8} \mathrm{RBC}$ are associated with a higher risk of leukopenia [22]. Interestingly, 6-TGN levels were far below $450 \mathrm{pmol} /$ $8 \times 10^{8}$ in all patients in the low-activity group, the maximum 6-TGN level being $171 \mathrm{pmol} / 8 \times 10^{8} \mathrm{RBC}$. At the same time, the low-activity group showed highest hematopoietic toxicity. Ten of 26 6-TGN results in the low-activity group were performed while patients had grade $3 / 4$ leukopenia or thrombocytopenia. These findings in the low-activity group imply the possibility that bone marrow suppression can result even from low levels of 6-TGN in patients with NUDT15 variants. Hematopoietic toxicity could not be antic- 
ipated by 6-TGN level alone in patients with NUDT15 variants, and therefore, existing 6-MP dose guidelines for TPMT variants are not applicable to this population. Although DNA-TG may seem more appropriate because it is the final metabolite of 6-MP therapy and has been recently reported to be correlated with TGN, 6-TGN levels have been more extensively studied and widely used in the clinical setting to monitor dose escalation or de-escalation in patients receiving 6-MP therapy [27]. Additionally, DNA-TG still requires prospective validation [28]. The development of clinically available monitoring methods is required for patients with NUDT15 variants. The effort to improve clinical validation of DNA-TG could be useful.

Few studies have analyzed 6-TGN levels using whole blood from patients with ALL according to their NUDT15 diplotypes. A Japanese group briefly reported that TGN levels were lower in patients with NUDT15 variants [29]. However, our study was performed on a much larger scale. A total of 182 children were included in our study, compared to 55 children reported in their study. In addition, while they analyzed two TGN results from a patient with high-risk, 26 results from five patients in the low-activity group were analyzed in this study.

In our study, patients with NUDT15 variants frequently experienced more serious bone marrow suppression and febrile neutropenia after administration of 6-MP, and showed serious 6-MP intolerance. The adverse effects and 6-MP intolerance got more severe as the number of variant alleles increased. Several previous reports have demonstrated thiopurine intolerance in patients with ALL with NUDT15 variants. Patients with NUDT15 variants in both alleles have been reported to use an extremely low dose of 6-MP, consistent with our results $[17,18]$. Clinical and laboratory information in this study were comprehensively collected during a year of maintenance therapy, which is a relatively longer study duration compared to other reports. Moriyama et al. [18] used the tolerated MP dose to compare thiopurine intolerance between groups according to NUDT15 diplotype. Tolerated MP dose was defined as the average of at least 14 daily doses after at least nine weeks of maintenance therapy. Another study defined MP dose intensity as the ratio between clinically prescribed MP dose and protocol dose, and it was monitored on a monthly basis for the entire six-month duration of the study [17]. Since previous studies did not provide complete information on thiopurine intolerance, the daily dose of 6-MP administered during a period of 1 year was investigated in this study. All six alleged haplotypes from four coding variants were screened, which is distinct from many previous reports that investigated a single mutation of rs 116855232 , since other variants beyond rs116855232 have only been recently discovered [18].
There were some differences in clinical and laboratory results between the intermediate-activity and normal-activity groups. While the intermediate-activity group was administered a significantly lower daily 6-MP dose and experienced therapy interruption and leukopenia more frequently than the normal-activity group, the incidence of febrile neutropenia was not significantly different between these groups. Moreover, while the lowest WBC and platelet counts were significantly different between these two groups, the absolute neutrophil counts and hemoglobin levels were not significantly different between the two groups. Thus, it could be interpreted that patients with a wild-type NUDT15 allele could maintain a somewhat protective effect but they were not capable of preventing thiopurine toxicity with doses over a certain range. Compared to WBC counts or hemoglobin levels, platelet counts were most significantly different between groups, which indicated that megakaryopoiesis is the more sensitive to thiopurine in patients with NUDT15 variants.

Unlike TPMT variants, which are rare in Asian population, studies have shown that NUDT15 variants were mostly discovered in this population, particularly in East Asians [16-18,21,30]. In this study, $26.4 \%$ of the patients had variant alleles. Additionally, the composition of diplotypes in this study was similar to that in 1000 Genomes Project and a recent Korean study [21]. rs116855232, which was the first reported NUDT15 variant, was verified to be the most common variant. Patients with wild-type NUDT15 were administered a mean daily $6-\mathrm{MP}$ dose of $31.1 \mathrm{mg} / \mathrm{m}^{2} /$ day, which was a substantially lower dose than the planned dose in the majority of ALL treatment protocols. A similar observation was made in other studies [31]. A previous study reported that East Asian genetic ancestry remained associated with 6-MP dose intensity after adjusting for rs116855232 [17], suggesting that there may be other undiscovered genetic or environmental factors affecting Asians. Kim et al. [32], recently reported that APEX1 rs2307486 variants conferred an increased risk of mercaptopurine-related early onset neutropenia in Korean pediatric ALL patients.

Even though this was a retrospective analysis, this study aimed to exclude possible compounding factors. Patients with TPMT variants were excluded from this study before analysis. 6-MP doses during the initial 3 months were excluded when comparing the 6-MP doses between the groups because 6-MP doses were frequently altered during the initial period. Demographic characteristics were not different between the groups. A limitation of this study was that it did not evaluate other anti-neoplastic agents used during maintenance therapy, such as methotrexate or vincristine.

It remains unclear whether NUDT15 genotype affects survival in children with ALL. Even though this study did not analyze relapse or survival, irrelevance between them was 
suggested because survival rates were not significantly different according to NUDT15 genotype in previous reports [11]. However, most of the earlier reports evaluated only a few patients with NUDT15 variants [31]. A previous COG study about thiopurines and risk of relapse in children with ALL demonstrated that 6-MP non-adherence and high intraindividual variability in TGN levels contributes to increased relapse risk [11]. Since patients with NUDT15 variants are exposed to frequent therapy interruptions, which can cause variability in TGN levels, NUDT15 genotype should be screened before 6-MP administration. 6-MP dose should be carefully administered and altered in high-risk populations. Further extensive studies on the impact of NUDT15 variants on treatment outcomes are required.

In conclusion, 6-TGN levels were substantially low in patients with NUDT15 variants who showed highest hematopoietic toxicity. The low ratio of 6-TGN level to 6-MP dose in patients with NUDT15 variants seems to be caused by enhanced incorporation of active thiopurine metabolites into DNA. With a known high incidence of NUDT15 variants in East Asia, NUDT15 genotype should be identified before thiopurine is administered. Thiopurine dose adjustment demands great caution in patients with NUDT15 variants regardless of their 6-TGN levels. Further studies are required for validating thiopurine dose guidelines in this population.

\section{Conflicts of Interest}

Conflict of interest relevant to this article was not reported.

\section{Acknowledgments}

This study was supported by a grant from the National R\&D Program for Cancer Control, Ministry of Health and Welfare, Republic of Korea (1420250) and a grant by the Korean Foundation for Cancer Research (KFCR-2017-D-1).

\section{References}

1. Pui CH, Carroll WL, Meshinchi S, Arceci RJ. Biology, risk stratification, and therapy of pediatric acute leukemias: an update. J Clin Oncol. 2011;29:551-65.

2. Lilleyman JS, Lennard L. Mercaptopurine metabolism and risk of relapse in childhood lymphoblastic leukaemia. Lancet. 1994;343:1188-90.

3. Childhood ALL Collaborative Group. Duration and intensity of maintenance chemotherapy in acute lymphoblastic leukaemia: overview of 42 trials involving 12000 randomised children. Lancet. 1996;347:1783-8.

4. Vora A, Goulden N, Wade R, Mitchell C, Hancock J, Hough $\mathrm{R}$, et al. Treatment reduction for children and young adults with low-risk acute lymphoblastic leukaemia defined by minimal residual disease (UKALL 2003): a randomised controlled trial. Lancet Oncol. 2013;14:199-209.

5. Fotoohi AK, Coulthard SA, Albertioni F. Thiopurines: factors influencing toxicity and response. Biochem Pharmacol. 2010;79:1211-20.

6. Schmiegelow K, Nielsen SN, Frandsen TL, Nersting J. Mercaptopurine/Methotrexate maintenance therapy of childhood acute lymphoblastic leukemia: clinical facts and fiction. J Pediatr Hematol Oncol. 2014;36:503-17.

7. Bhatia S, Landier W, Hageman L, Kim H, Chen Y, Crews KR, et al. 6MP adherence in a multiracial cohort of children with acute lymphoblastic leukemia: a Children's Oncology Group study. Blood. 2014;124:2345-53.

8. Takatsu N, Matsui T, Murakami Y, Ishihara H, Hisabe T, Nagahama T, et al. Adverse reactions to azathioprine cannot be predicted by thiopurine S-methyltransferase genotype in Japanese patients with inflammatory bowel disease. J Gas- troenterol Hepatol. 2009;24:1258-64.

9. Koren G, Ferrazini G, Sulh H, Langevin AM, Kapelushnik J, Klein J, et al. Systemic exposure to mercaptopurine as a prognostic factor in acute lymphocytic leukemia in children. $\mathrm{N}$ Engl J Med. 1990;323:17-21.

10. Relling MV, Hancock ML, Boyett JM, Pui CH, Evans WE. Prognostic importance of 6-mercaptopurine dose intensity in acute lymphoblastic leukemia. Blood. 1999;93:2817-23.

11. Bhatia S, Landier W, Hageman L, Chen Y, Kim H, Sun CL, et al. Systemic exposure to thiopurines and risk of relapse in children with acute lymphoblastic leukemia: a Children's Oncology Group Study. JAMA Oncol. 2015;1:287-95.

12. Lennard L, Lilleyman JS, Van Loon J, Weinshilboum RM. Genetic variation in response to 6-mercaptopurine for childhood acute lymphoblastic leukaemia. Lancet. 1990;336:225-9.

13. Evans WE, Hon YY, Bomgaars L, Coutre S, Holdsworth M, Janco R, et al. Preponderance of thiopurine S-methyltransferase deficiency and heterozygosity among patients intolerant to mercaptopurine or azathioprine. J Clin Oncol. 2001;19: 2293-301.

14. Relling MV, Gardner EE, Sandborn WJ, Schmiegelow K, Pui $\mathrm{CH}$, Yee SW, et al. Clinical pharmacogenetics implementation consortium guidelines for thiopurine methyltransferase genotype and thiopurine dosing: 2013 update. Clin Pharmacol Ther. 2013;93:324-5.

15. Swen JJ, Nijenhuis M, de Boer A, Grandia L, Maitland-van der Zee AH, Mulder $\mathrm{H}$, et al. Pharmacogenetics: from bench to byte: an update of guidelines. Clin Pharmacol Ther. 2011;89: 662-73.

16. Yang SK, Hong M, Baek J, Choi H, Zhao W, Jung $\mathrm{Y}$, et al. A 
common missense variant in NUDT15 confers susceptibility to thiopurine-induced leukopenia. Nat Genet. 2014;46: 1017-20.

17. Yang JJ, Landier W, Yang W, Liu C, Hageman L, Cheng C, et al. Inherited NUDT15 variant is a genetic determinant of mercaptopurine intolerance in children with acute lymphoblastic leukemia. J Clin Oncol. 2015;33:1235-42.

18. Moriyama T, Nishii R, Perez-Andreu V, Yang W, Klussmann FA, Zhao X, et al. NUDT15 polymorphisms alter thiopurine metabolism and hematopoietic toxicity. Nat Genet. 2016;48: 367-73.

19. Zgheib NK, Akika R, Mahfouz R, Aridi CA, Ghanem KM, Saab R, et al. NUDT15 and TPMT genetic polymorphisms are related to 6-mercaptopurine intolerance in children treated for acute lymphoblastic leukemia at the Children's Cancer Center of Lebanon. Pediatr Blood Cancer. 2017;64:146-50.

20. Takagi Y, Setoyama D, Ito R, Kamiya H, Yamagata $Y$, Sekiguchi M. Human MTH3 (NUDT18) protein hydrolyzes oxidized forms of guanosine and deoxyguanosine diphosphates: comparison with MTH1 and MTH2. J Biol Chem. 2012;287:21541-9.

21. Kim HT, Choi R, Won HH, Choe YH, Kang B, Lee K, et al. NUDT15 genotype distributions in the Korean population. Pharmacogenet Genomics. 2017;27:197-200.

22. Dervieux T, Meyer G, Barham R, Matsutani M, Barry M, Boulieu R, et al. Liquid chromatography-tandem mass spectrometry analysis of erythrocyte thiopurine nucleotides and effect of thiopurine methyltransferase gene variants on these metabolites in patients receiving azathioprine/6-mercaptopurine therapy. Clin Chem. 2005;51:2074-84.

23. Lee MN, Kang B, Choi SY, Kim MJ, Woo SY, Kim JW, et al. Relationship between azathioprine dosage, 6-thioguanine nucleotide levels, and therapeutic response in pediatric patients with IBD treated with azathioprine. Inflamm Bowel Dis. 2015;21:1054-62.

24. Lee MN, Kang B, Choi SY, Kim MJ, Woo SY, Kim JW, et al. Impact of genetic polymorphisms on 6-thioguanine nucleotide levels and toxicity in pediatric patients with IBD treated with azathioprine. Inflamm Bowel Dis. 2015;21:2897-908.

25. Lennard L, Welch JC, Lilleyman JS. Thiopurine drugs in the treatment of childhood leukaemia: the influence of inherited thiopurine methyltransferase activity on drug metabolism and cytotoxicity. Br J Clin Pharmacol. 1997;44:455-61.

26. Valerie NC, Hagenkort A, Page BD, Masuyer G, Rehling D, Carter M, et al. NUDT15 hydrolyzes 6-thio-deoxyGTP to mediate the anticancer efficacy of 6-thioguanine. Cancer Res. 2016;76:5501-11.

27. Ebbesen MS, Nersting J, Jacobsen JH, Frandsen TL, Vettenranta $\mathrm{K}$, Abramsson J, et al. Incorporation of 6-thioguanine nucleotides into DNA during maintenance therapy of childhood acute lymphoblastic leukemia-the influence of thiopurine methyltransferase genotypes. J Clin Pharmacol. 2013;53: 670-4.

28. Nielsen SN, Grell K, Nersting J, Frandsen TL, Hjalgrim LL, Schmiegelow K. Measures of 6-mercaptopurine and methotrexate maintenance therapy intensity in childhood acute lymphoblastic leukemia. Cancer Chemother Pharmacol. 2016;78: 983-94.

29. Moriyama T, Nishii R, Lin TN, Kihira K, Toyoda H, Jacob N, et al. The effects of inherited NUDT15 polymorphisms on thiopurine active metabolites in Japanese children with acute lymphoblastic leukemia. Pharmacogenet Genomics. 2017;27: 236-9.

30. Kham SK, Soh CK, Liu TC, Chan YH, Ariffin H, Tan PL, et al Thiopurine S-methyltransferase activity in three major Asian populations: a population-based study in Singapore. Eur J Clin Pharmacol. 2008;64:373-9.

31. Tanaka Y, Kato M, Hasegawa D, Urayama KY, Nakadate H, Kondoh K, et al. Susceptibility to 6-MP toxicity conferred by a NUDT15 variant in Japanese children with acute lymphoblastic leukaemia. Br J Haematol. 2015;171:109-15.

32. Kim H, Seo H, Park Y, Min BJ, Seo ME, Park KD, et al. APEX1 Polymorphism and mercaptopurine-related early onset neutropenia in pediatric acute lymphoblastic leukemia. Cancer Res Treat. 2017 Sep 4 [Epub]. https://doi.org/10.4143/crt. 2017.351. 